\title{
ON THE EVALUATION OF THE GRADIENT TREE BOOSTING MODEL FOR GROUNDWATER LEVEL FORECASTING
}

\author{
Sujay Raghavendra Naganna ${ }^{1}$, Beste Hamiye Beyaztas ${ }^{2}$, Neeraj Dhanraj Bokde ${ }^{3, *}$ and Asaad M. \\ Armanuos $^{4}$ \\ ${ }^{1}$ Department of Civil Engineering, Siddaganga Institute of Technology, Tumakuru 572103, Karnataka, India. \\ ${ }^{2}$ Department of Statistics at Istanbul Medeniyet University, Turkey. \\ ${ }^{3}$ Department of Engineering - Renewable Energy and Thermodynamics, Aarhus University, 8000, Denmark. \\ ${ }^{4}$ Irrigation and Hydraulics Engineering Department, Civil Engineering Department, Faculty of Engineering, Tanta \\ University, Egypt. \\ *Corresponding: neerajdhanraj@eng.au.dk
}

\begin{abstract}
Though groundwater is a replenishable resource, it's over exploitation has posed greater problem of its depletion. Hence, monitoring and forecasting of groundwater levels has become a primary task of governmental water boards/agencies for sustainable water management. The current study focused on evaluating the performance of Gradient Tree Boosting (GTB) model with that of conventional Adaptive Neuro-Fuzzy Inference System (ANFIS) and Group Method of Data Handling (GMDH) models in forecasting groundwater levels of two coastal aquifers. Data of two groundwater level monitoring wells penetrating into unconfined aquifers located at Shirtadi and Rayee near to Mangalore city of Karnataka state, India was considered in the present study. Monthly groundwater level data of the years 2000 - 2013 were used for model simulation; wherein $70 \%$ of data was used for model training and the remaining $30 \%$ served as testing data. Comparative result evaluation shows that the proposed GTB approach for one month ahead groundwater level forecasting was giving much accurate results than the other models for the same period of time and same set of data. For Rayee monitoring well, the error statistic, RRMSE of GTB, GMDH and ANFIS models obtained during test phase were $0.473,0.517$ and 0.7522 , respectively. The comparison is examined further with different performance metrics.
\end{abstract}

Keywords: ANFIS model; forecasting; groundwater level; Gradient Tree Boosting; GMDH.

\section{Introduction}

One of the prominent water resources on earth is groundwater [1]. Groundwater is mainly used for irrigation purposes as it accounts for almost $80 \%$ of groundwater usage. Groundwater is also used for domestic, industrial, and drinking purposes. Groundwater level prediction is important for effective management of ground water resources [2], [3]. The variations in groundwater level is influenced by time-dependent recharging and discharge processes, tidal effects, streamflow variations, climate change and weather impacts [4].

Recently, scholars have relied on computational modelling of flow and transport to understand the hydrology of water bodies and groundwater [5], [6]. However, the conversion of the physical processes into mathematical formulations, as well as the lack of sufficient data to execute the modelling process, is some of the challenges of using numerical models. Interestingly, artificial modelling techniques have been developed over the last two decades as an approach to overcome these shortcomings of the numerical models [7]-[9]. For instance, artificial neural network (ANN) model has been used for the prediction of fluctuations in groundwater level; the study relied on time-lagged water levels as the model input [10]. Furthermore, the ANN model was improved by Jalalkamali and Jalalkamali [11] through merging it with the genetic algorithm (GA) in order to address some of the common issues of the conventional ANN, such as optimizing the process of finding the global minimum of the error cost function. The performance of ANN and support vector machine (SVM) models in groundwater levels prediction has been compared by Yoon et al. [12]; while genetic programming (GP) and adaptive neural fuzzy inference system (ANFIS) were investigated as artificial tools for groundwater levels prediction in 3 observation wells in the Karaj plain of Iran [13]. The study by Shiri et al. (2013) focused on the performance of various data mining techniques in the prediction of groundwater level while support vector machines (SVM) were used to model the groundwater level of Hamadan-Bahar plain, west of Iran [14]. The use of ANN for groundwater level prediction under the impact of different factors for the period of 20002015 has been conducted for Vietnam region and evidenced its capacity [15]. 
ANN and M5 Tree models have been used by Kaya et al. [16] for groundwater level prediction in the Reyhanli region of Turkey and the study found both models to have performed equally in groundwater level prediction. Lee et al. [17] investigated the performance of ANN model in the prediction of groundwater level in Yangpyeong riverside, South Korea; the input variables used in the study consisted of one natural factor and two artificial indicators. A two phase data-driven model has been used for time series groundwater level prediction [18]; the study relied on spatial-temporal analysis and least-square SVM (LS-SVM) but found better performance with the LS-SVM model compared to the other models used. The groundwater level prediction performance of ANN, GP, SVM, and ELM at six locations in the district of Vizianagaram, Andhra Pradesh, has been compared by Natarajan and Sudheer [19]; the study observed the best performance with ELM compared to the ANN, GP, and SVM models. In another study, the prediction performance of ensemble model (EANNGA), emotional ANN (EANN), generalized regression neural network (GRNN), and the conventional feedforward neural network (FFNN) in the prediction of one-month ahead groundwater level has been compared by Roshni et al. [20] in a coastal aquifer system. From the results, the EANN-GA model performed better than the EANN, FFNN, and GRNN models. The study by Rahman et al. [21] evaluated the performance of hybridized machine learning-wavelet transform model in the prediction of groundwater level; the outcome of the study demonstrated the possibility of hybridizing some novel ML methods, such as Random Forests and eXtreme Gradient Boosting with wavelet transforms for accurate prediction of groundwater level in Southern Japan. Recent researches include, development of robust hybrid ML models such as the multilayer perceptron neural network optimized by whale algorithm (MLP-WA), for groundwater level predictions in the Yazd province of Iran [8]; ensemble modelling framework for groundwater level prediction in urban areas (Bangalore) of India [22]. The groundwater dynamics changes from region to region based on climate and rainfall pattern over the area. The highly diversified occurrence, wide variations in the precipitation characteristics and seasonal variations in utilization of ground water makes its management a challenging task. Therefore, it becomes pertinent to analyze and understand the groundwater fluctuations in different hydrogeological situations [23]. Hence, the current study focused on modeling groundwater levels of two shallow coastal aquifers by the development of novel Gradient Tree Boosting (GTB) model and compare its performance against conventional Adaptive Neuro-Fuzzy Inference System (ANFIS) and Group Method of Data Handling (GMDH) models.

The remainder of this article is organized as follows: Section 2 demonstrates the forecasting models that are employed in the case study. Section 3 describes the case study and methodology wherein geological features of two groundwater level monitoring wells penetrating into unconfined aquifers located at Shirtadi and Rayee near to Mangalore city of Karnataka state, India have been presented. In Section 4, the results and discussions of the case study are presented. Finally, the conclusions of the paper are summarized in Section 5.

\section{Applied machine learning models}

\subsection{Adaptive Neuro-Fuzzy Inference System (ANFIS)}

ANFIS model that consist of ANN and Fuzzy Inference System (FIS) was proposed by Jang (1993) [24]. Both models were used as a multilayer network in ANFIS for mapping and modelling of the input-output relationship [25] while the rules are applied as membership functions (MFs) for a fuzzy decision. The FIS relies on the IFTHEN principle; hence, the FIS can be used as a reliable prediction model in situations where there is uncertainty in the dataset. Figure 1 shows the architecture of ANFIS. The adaptive nodes in the first layer are designed for input membership grades generation with the following output:

$$
\begin{aligned}
& O_{1, i}=\mu_{A i}(x) \text { for } i=1,2 \\
& O_{1, i}=\mu_{B i-2}(y) \text { for } i=3,4
\end{aligned}
$$

where, the input nodes are represented by $\mathrm{x}$ and $\mathrm{y}$ while $A_{i}$ and $B_{i}$ are the fuzzy set; $\mu(x)$ and $\mu(y)$ represent the MFs (there are different shapes of the MFs, such as triangular, general bell, trapezoidal, and Gaussian). The nodes in the second layer are designated as $\Pi$, with all the incoming signals being multiplied by each node. The firing strength of each rule is described by the output; this can be calculated thus:

$$
O_{2, i}=w_{i}=\mu_{A i}(x) * \mu_{B i}(y) \text { for } i=1,2
$$

The nodes in the third layer are marked as $N$; the normalized firing strength in this layer is computed using the following equation. The ith rule contribution of each node is calculated in the fourth layer while the total output of the ANFIS is determined in the fifth layer.

$$
O_{3, i}=\bar{w}_{i}=\frac{w_{i}}{w_{1}+w_{2}} \quad \text { for } i=1,2
$$




$$
\begin{gathered}
O_{4, i}=\bar{w}_{i} \times f_{i} \\
O_{5, i}=\sum_{i=1}^{n} \bar{w}_{i} . f_{i}
\end{gathered}
$$

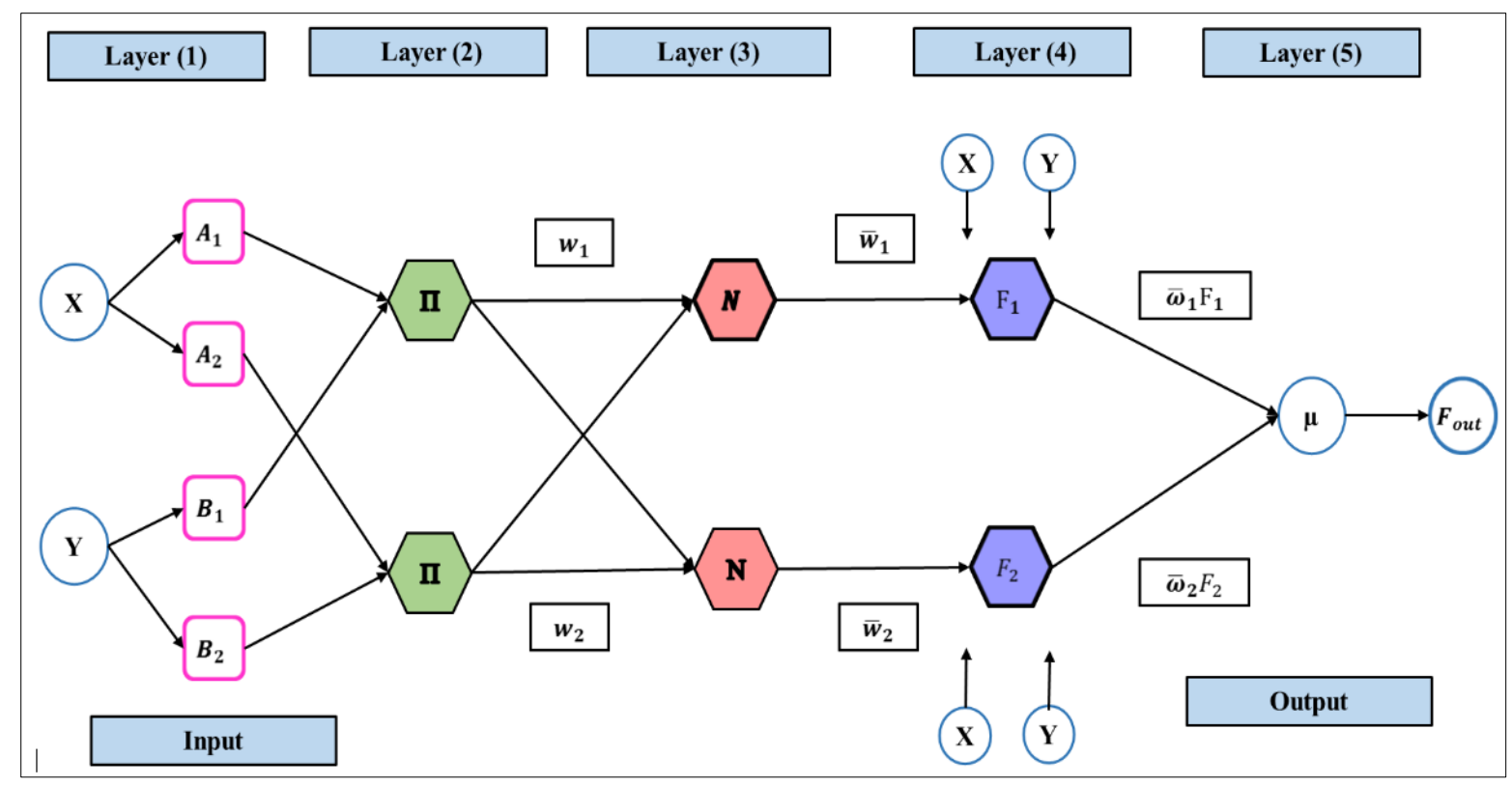

Figure 1: Structure of ANFIS model.

\subsection{Group Method of Data Handling (GMDH)}

Ivakhnenko, a former Soviet scientist, first suggested the GMDH algorithm as a method for the identification of the nonlinear input-out relationships [26]. This model generates a high-order polynomial network which in principle is a feed-forward \& multilayer neural network (Figure 2). It provides a platform for self-organized data mining wherein the modelling variables, parameters, and structure are automatically determined [27]. In the GMDH models, the input dataset is partitioned into 2 groups, with the first group being used for the approximation of the parameters of each neuron; this aids in partial description of the modelling process. The second group of dataset is for the determination of the performance of each of the models that efficiently described the process [28]. The training dataset is specifically used to approximate the Kolmogorov-Gabor polynomial coefficients while the testing set is used for error evaluation in the GMDH network. In the GMDH network, successive layers are constructed and the connections between the layers represent the individual terms of a polynomial [29]. An external criterion is used to assess and evaluate the output of each neuron; neurons that performed poorly are eliminated by the model while the ones that performed excellently are retained in the next layer. New layers ae created by repeating this step until there is stability in the error criterion. Previous studies have provided detailed description of the GMDH model [29]-[31]. The Volterra functional series of the Kolmogorov-Gabor polynomial expresses the general input-output relationship in the GMDH algorithm as follows [32]:

$$
y(t)=a_{0}+\sum_{i=1}^{n} a_{i} x_{i}+\sum_{i=1}^{n} \sum_{j=1}^{n} a_{i j} x_{i} x_{j}+\sum_{i=1}^{n} \sum_{j=1}^{n} \sum_{k=1}^{n} a_{i j k} x_{i} x_{j} x_{k}
$$

where, $y(t)$ represent the output variable, while $X\left(x_{1}, x_{2}, \ldots, x_{n}\right)$ represent the input variable's vector; A $\left(a_{1}, a_{2}, \ldots, a_{i}\right)$ represent the vector weights or coefficients. In the GMDH model, variables with the most influence on the system are first paired and set a default threshold; this effectively eliminates the poorly performing variables. 


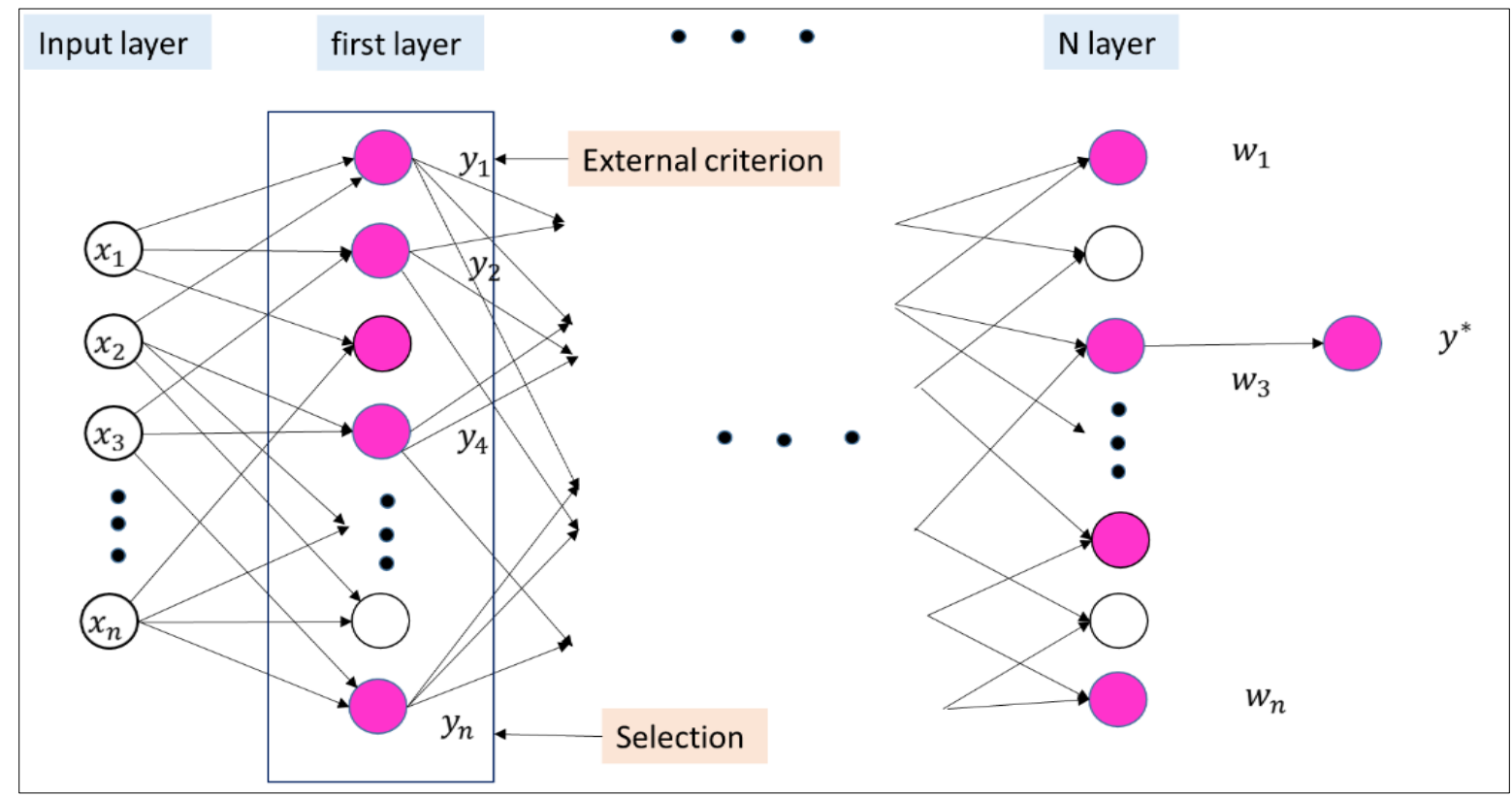

Figure 2: Structure of GMDH model.

\subsection{Gradient Tree Boosting (GTB)}

Let $X=\left\{x_{1}, \ldots, x_{n}\right\}$ be the vector input with output as $\mathrm{y}$; the training process of a metamodel is mainly aimed at establishing a relationship which can rely on a given training data set, $\left\{X_{i}, y_{i}\right\}_{1}^{N}$ to represent a function $F_{\text {opt }}(X)$ and map $\mathrm{X}$ to $\mathrm{y}$ in a way that the predetermined loss function, $\operatorname{Loss}(\mathrm{y}, \mathrm{F}(\mathrm{X}))$, of the expected value $F\left(X_{i}\right)$ and the exact value $y_{i}$ is minimized. Hence, $F_{\text {opt }}(X)$ can be expressed thus:

$$
F_{\text {opt }}(X)=\arg _{F(X)} \min \operatorname{Loss}(y, F(X))
$$

The solution to the problem in Eq. (8) is achieved by developing a tree ensemble model that sequentially combined several decision trees (also called base learners) to achieve a stronger learner. Being that a base learner can only predict output with a high residual, it is also called is a weak base learner. The obtained residual from the previous decision tree (DT) is used at each iteration as the output for the development of new DTs [33]; this improves the accuracy of the training process and reduces the residual. The output of the GTB method is calculated using a tree ensemble model as follows; where $\left\{\beta_{m}\right\}_{0}^{M}$ are added to accommodate the importance of the base learner [34].

$$
F(X)=\sum_{m=0}^{M} \beta_{m} f\left(X, a_{m}\right)
$$

where, $f\left(X, a_{m}\right)$ could represent simple functions of $X$ with a range of parameters $a_{m}\left\{a_{1}^{m}, a_{2}^{m}, \ldots, a_{n}^{m}\right\}$. Based on an initial guess $F_{0}(X)$, the equation for updating the model can be written as:

$$
F_{m}(X)=F_{m-1}(X)+\beta_{m} f\left(X, a_{m}\right)
$$

Simply, $F_{0}(X)$ can be set to 0 ; the two-step gradient decent-like procedure is used to determine $\beta_{m}$ and $a_{m}$; first, the following equation is used to calculate $a_{m}$ :

$$
a_{m}=\arg _{a, \rho} \min \sum_{i=1}^{N}\left[\bar{y}_{i m}-\rho f\left(X_{i} ; a\right)\right]^{2}
$$

where $\rho$ is defined as the step size and

$$
\bar{y}_{i m}=-\left[\frac{\partial \operatorname{Loss}\left(y_{i}, F\left(X_{i}\right)\right)}{\partial F\left(X_{i}\right)}\right]_{F(X)=F_{m-1}(X)}
$$

Then, $\beta_{m}$ is calculated as: 


$$
\beta_{m}=\arg _{\beta} \min \sum_{i=1}^{N} \operatorname{Loss}\left(y_{i}, F_{m-1}\left(X_{i}\right)+\beta f\left(X_{i} ; a\right)\right)
$$

\section{Case study and modeling development}

Data of two groundwater level monitoring wells penetrating into unconfined aquifers located at Shirtadi and Rayee near to Mangalore city of Karnataka state, India was considered in the present study. The local geological features are of archaic origin characterized by exposed lateritic soil in hilly tracts and sandy soil. Uni-variate machine learning based time series models were implemented. Monthly groundwater level data of the years 2000 - 2013 were used for model simulation; wherein $70 \%$ of data was used for model training and the remaining $30 \%$ served as testing data. Table 1 presents the groundwater level data statistics of the monitoring wells. The input - output structure of the models developed were arrived based on Autocorrelation (ACF) and Partial Autocorrelation (PACF) plots as presented in Figures 3 and 4. For Shirthadi and Rayee monitoring well, both ACF and PACF patterns trailing off at several lags, 12, 11, 10, 1 and 0 . This ensures that the time series have significant series correlations, and an effective model can be designed. The input - output structure of models for one month ahead groundwater level forecast is as presented below.

$$
\mathrm{GWT}_{(\mathrm{t}-12)}+\mathrm{GWT}_{(\mathrm{t}-1)}+\mathrm{GWT}_{(\mathrm{t}-10)}+\mathrm{GWT}_{(\mathrm{t}-1)}+\mathrm{GWT}_{(\mathrm{t})} \longrightarrow \mathrm{GWT}_{(\mathrm{t}+1)}
$$
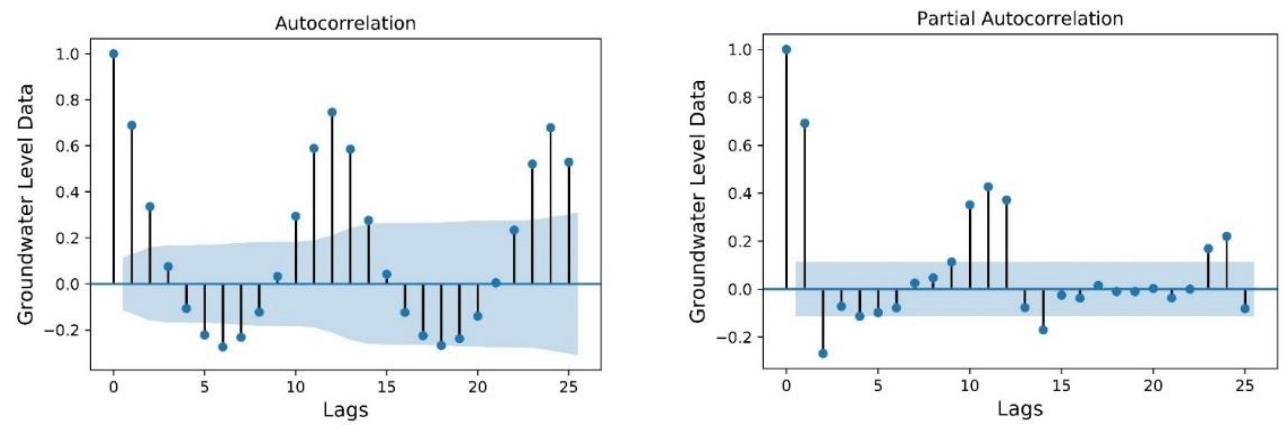

Figure 3: Autocorrelation and Partial autocorrelation in Groundwater level data of Shirthadi monitoring well.
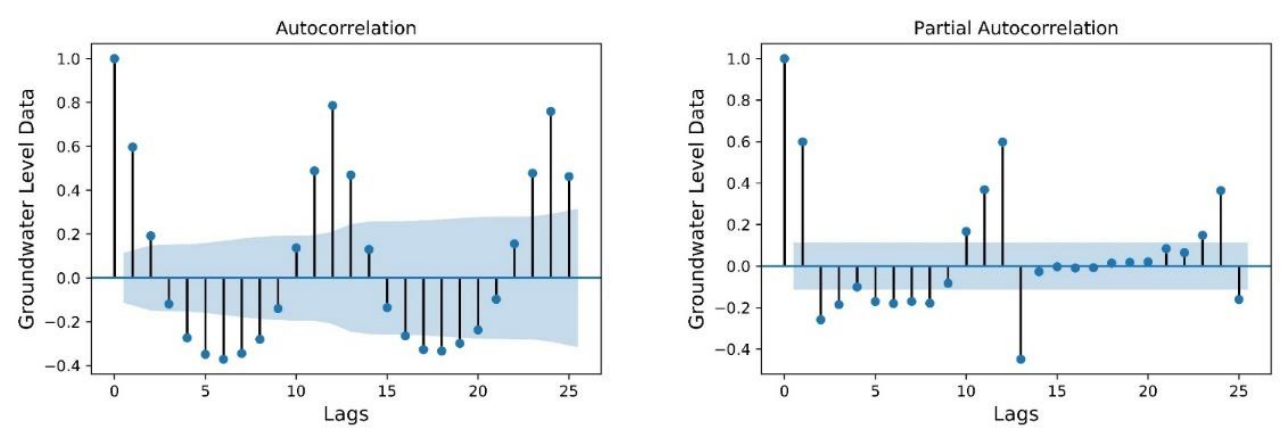

Figure 4: Autocorrelation and Partial autocorrelation in Groundwater level data of Rayee monitoring well.

Table 1: Groundwater level Data Statistics of Monitoring wells.

\begin{tabular}{cccccc}
\hline $\begin{array}{c}\text { Monitoring } \\
\text { Well }\end{array}$ & Minimum & Maximum & Mean & $\begin{array}{c}\text { Standard } \\
\text { Deviation }\end{array}$ & $\begin{array}{c}\text { Coefficient of } \\
\text { Variation }\end{array}$ \\
\hline Shirthadi & 13.35 & 0.68 & 8.960 & 2.835 & 0.316 \\
\hline Rayee & 12.4 & 1.03 & 7.022 & 2.779 & 0.396 \\
\hline
\end{tabular}

Note: Groundwater level data is the depth to water table below ground level in meters.

The forecast performance of GTB, GMDH and ANFIS models were evaluated using statistical indices as mentioned below.

i. $\quad$ Relative Root Mean Square Error (RRMSE) [35]: 


$$
R M S E=\sqrt{\frac{\sum_{i=1}^{N}\left(O_{i}-P_{i}\right)^{2}}{N}} ; \text { and } R R M S E=\frac{R M S E}{\sigma_{o b s}}
$$

ii. Normalized Nash Sutcliffe Efficiency (NNSE) [36]:

$$
N S E=1-\frac{\sum_{i=1}^{N}\left(P_{i}-O_{i}\right)^{2}}{\sum_{i=1}^{N}\left(O_{i}-\bar{O}\right)^{2}} ; \text { and NNSE }=\frac{1}{2-N S E}
$$

iii. $\quad$ Normalized Mean Bias (NMB) [37]:

$$
N M B=\frac{\sum_{i=1}^{N}\left(P_{i}-O_{i}\right)}{\sum_{i=1}^{N} O_{i}}
$$

iv. Kling Gupta Efficiency (KGE) [38]:

$$
K G E=1-\sqrt{(R-1)^{2}+(\beta-1)^{2}+(\gamma-1)^{2}}
$$

where, Correlation Coefficient, Bias ration and variability

$$
R=\frac{\sum_{i=1}^{N}\left(O_{i}-\bar{O}\right) \cdot\left(P_{i}-\bar{P}\right)}{\sqrt{\sum_{i=1}^{N}\left(O_{i}-\bar{O}\right)^{2} \cdot \sum_{i=1}^{N}\left(P_{i}-\bar{P}\right)^{2}}}, \beta=\frac{\bar{P}}{\bar{O}}, \gamma=\frac{C V_{P}}{C V_{O}}=\frac{\sigma_{p} / \bar{P}}{\sigma_{o} / \bar{O}}
$$

v. Willmott's Index of Agreement (WI) [39]:

$$
W I=1-\frac{\sum_{i=1}^{N}\left[\left(P_{i}-\bar{O}\right)-\left(O_{i}-\bar{O}\right)\right]^{2}}{\sum_{i=1}^{N}\left[\left(P_{i}-\bar{O}\right)+\left(O_{i}-\bar{O}\right)\right]^{2}}
$$

where, $O$ and $P$ represent the observed and predicted groundwater level values, respectively. $\bar{O}$ and $\bar{P}$ are the mean values of observed and forecasted time-series, $\sigma_{o}$ and $\sigma_{p}$ are the standard deviation values of observed and forecasted time-series, respectively. 'N' represents the total number of data samples.

\section{Results and Discussion}

Groundwater level or water table is a key indicator for the water scientists and hydro-geologists worldwide, to monitor or estimate groundwater reserves so as to predict future droughts and famine conditions. Herein, the implemented techniques (GTB, GMDH and ANFIS) function as forecasting models to predict the values of groundwater levels of subsequent month using the antecedent time-series. Many researchers have proposed different machine learning and hybrid algorithms to forecast multi-scale groundwater levels. But in the current scenario, a supervised machine learning technique, Gradient Tree Boosting commonly known as GTB, which are ensembles of so-called decision trees is used for forecasting to get high precision results. The performance and memory requirements of GTB models are dependent on optimal tuning of its hyper-parameters namely, the number of boosting stages, learning rate, maximum depth, and the loss function which were found based on trial and error approach. Similarly, the model parameters of GMDH and ANFIS models were also worked out by trial and error approach.

Table 2 presents the performance statistics of machine learning models in groundwater level forecasting of Shirthadi monitoring well. The performance of our novel model i.e., GTB is compared with that of the widely used GMDH and ANFIS models. The error statistic, RRMSE of GTB, GMDH and ANFIS models obtained during test phase were $0.473,0.517$ and 0.7522 , respectively. It could be observed that the ANFIS model performed poorly with maximum error. NMB statistic indicates whether the modeled mean under or overestimates the observed mean. The NMB values close to 0 shows the model superiority. From, the Table 2 it's evident that GTB performed better than GMDH and ANFIS models based on all the five evaluation indices. In terms of $\mathrm{R}^{2}$ statistic obtained in scatter plots (Figure 5), GTB gives the best fit with minimum over-fitting compared to the GMDH and ANFIS forecasts.

Table 2. Performance of ML models in GW level forecasting of Shirthadi Monitoring Well

\begin{tabular}{lccc}
\hline Statistic & GTB & GMDH & ANFIS \\
\hline KGE & 0.8470 & 0.8167 & 0.6908 \\
NMB & -0.0020 & 0.0117 & 0.0392 \\
NNSE & 0.8153 & 0.7871 & 0.6358 \\
RRMSE & 0.4730 & 0.5170 & 0.7522 \\
WI & 0.9354 & 0.9194 & 0.8379 \\
\hline
\end{tabular}




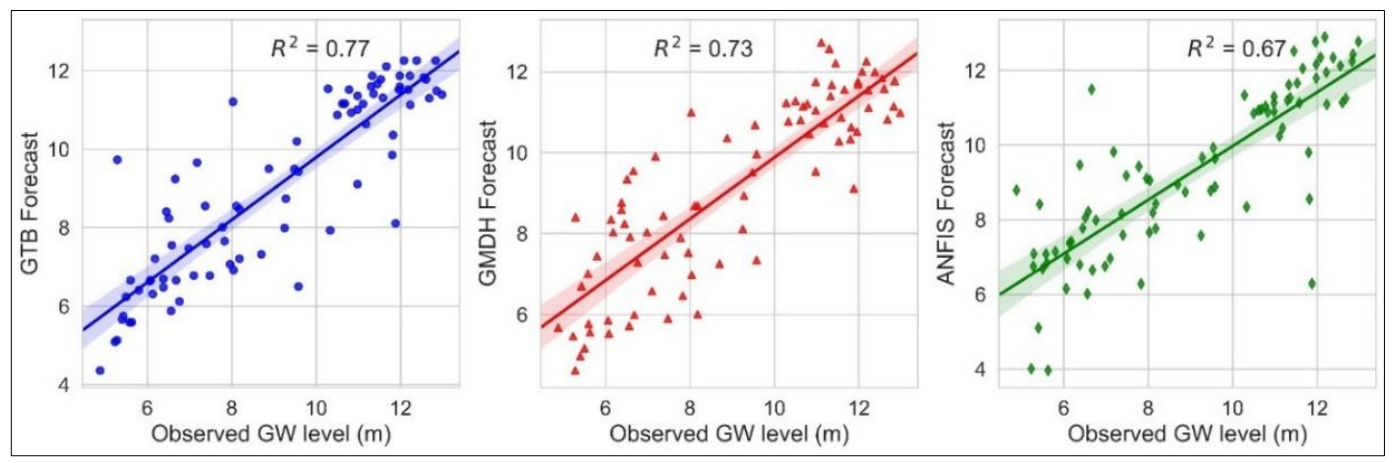

Figure 5: Scatter plots of GTB, GMDH and ANFIS groundwater level forecasts of Shirthadi well.

The detailed performance metrics of test phase with respect to Rayee monitoring well are depicted in Table 3. Among the three different regressors, GTB provides prediction with minimum error (RRMSE) and maximum efficiency (NNSE/KGE/WI). In terms of RRMSE, GTB outperforms GMDH and ANFIS models by 51.68\% and $64.20 \%$, respectively. The NMB statistic of GTB is the minimum and this demonstrates the superiority of the GTB method. It's worth mentioning that the groundwater level forecast performance of GTB is significantly closer to the 1:1 line with $\mathrm{R}^{2}=0.95$ as indicated in the scatter plots depicted in Figure 6 . Usually, the problem of over-fitting is avoided by the usage of boosting algorithm like GTB. In GTB, each new tree aid in correcting errors of the previously trained tree and optimal combination of parameters assist to build more generalized models. From Table 2, its clearly evident that the efficiency (WI) of GTB model is higher by $1.74 \%$ and $11.63 \%$ to that of GMDH and ANFIS models, respectively.

Table 3. Performance of ML models in GW level forecasting of Rayee Monitoring Well.

\begin{tabular}{ccrc}
\hline Statistic & \multicolumn{1}{c}{ GTB } & \multicolumn{1}{c}{ GMDH } & \multicolumn{1}{c}{ ANFIS } \\
\hline KGE & 0.9642 & 0.8060 & 0.8086 \\
\hline NMB & -0.0143 & -0.0159 & 0.0312 \\
\hline NNSE & 0.9568 & 0.8379 & 0.7393 \\
\hline RRMSE & 0.2112 & 0.4371 & 0.5901 \\
\hline WI & 0.9883 & 0.9405 & 0.9020
\end{tabular}
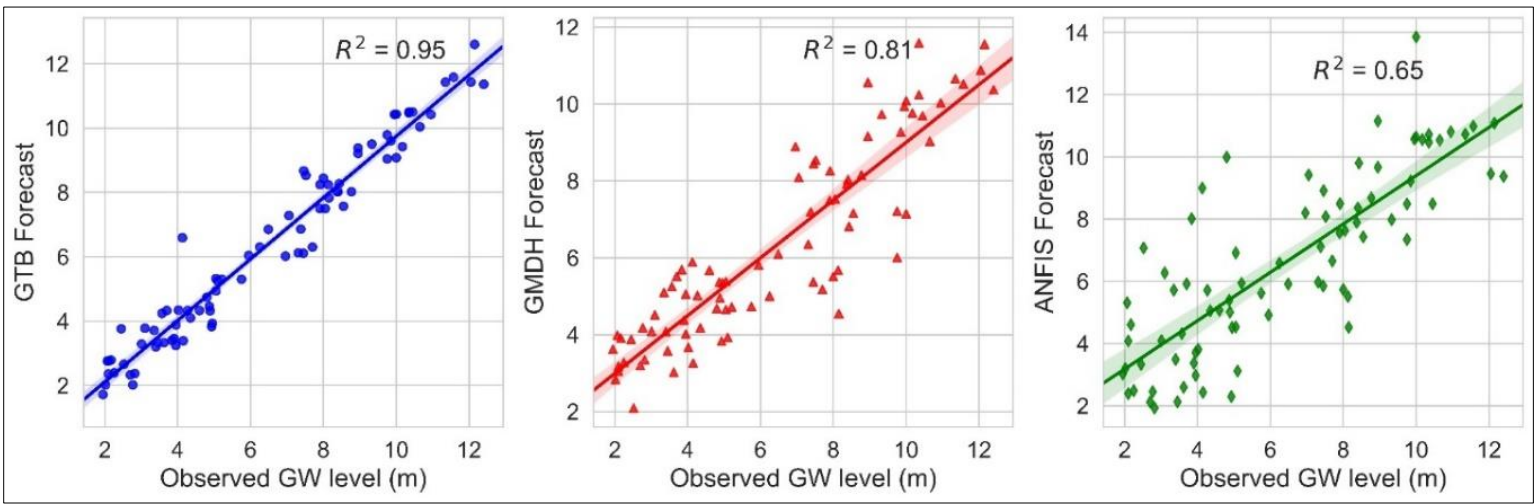

Figure 6: Scatter plots of GTB, GMDH and ANFIS groundwater level forecasts of Rayee well.

The Taylor diagrams supply visual analysis to rank the machine learning models based on three statistical indices (RMSD, Correlation coefficient and Standard deviation). For the models of Shirthadi and Rayee well locations, Taylor diagrams are presented in Figures 7 and 8, respectively. Well the Taylor diagrams indeed supplement the earlier evidences and portray that GTB regressor outperforms the other two namely, the GMDH and ANFIS in terms of all statistical indices. Comparative evaluation shows that the proposed GTB approach for one month ahead groundwater level forecasting was giving much accurate results than the other models for 
the same period of time and same set of data. The WI and NNSE indices also show the superiority of GTB model predictions. From Table 3, its clearly evident that the NNSE of GTB model is higher by $14.19 \%$ and $29.42 \%$ to that of GMDH and ANFIS models, respectively.

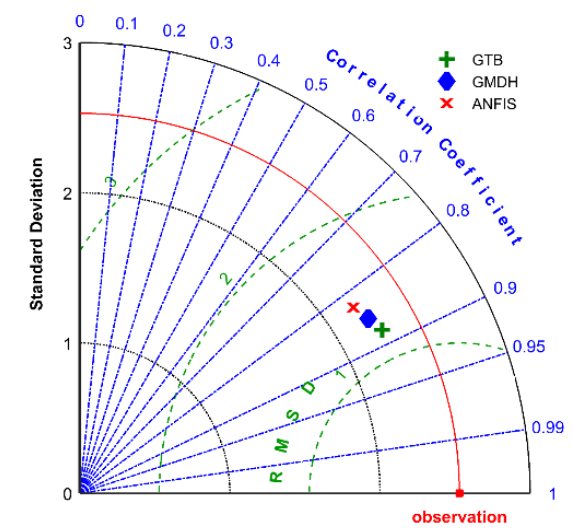

Figure 7: Taylor Diagram for comparative evaluation of performance of GTB, GMDH and ANFIS models in forecasting GW levels of Shirthadi monitoring well.

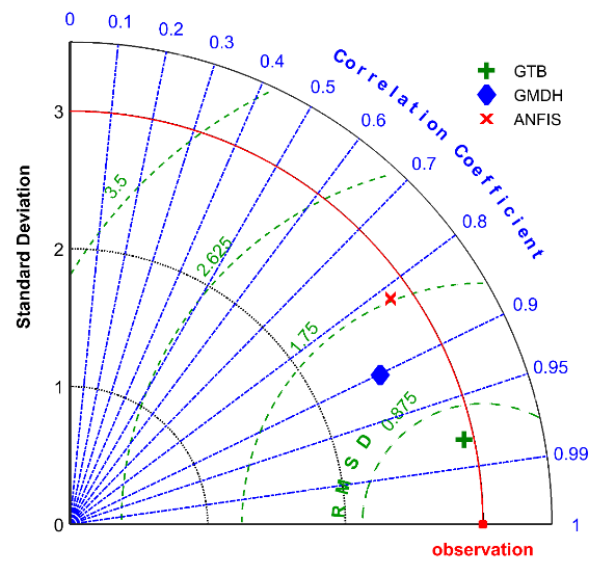

Figure 8: Taylor Diagram for comparative evaluation of performance of GTB, GMDH and ANFIS models in forecasting GW levels of Rayee monitoring well.

\section{Conclusions}

New methodologies for groundwater level forecasting along with the existing approaches was considered in the present study. The GMDH, ANFIS and GTB were implemented to check for the model suitability and applicability. Based on several statistical and visual evaluation metrics, GTB was found to provide better one month ahead groundwater level forecasts at both Shirthadi and Rayee locations considered. For Rayee monitoring well, the error statistic, RRMSE of GTB, GMDH and ANFIS models obtained during test phase were $0.473,0.517$ and 0.7522 , respectively. The comparison is examined further with different performance metrics. In addition, GTB is computationally efficient and fast for time series data prediction along with high prediction accuracy.

Acknowledgement(s): The authors would like to thank the Department of Mines and Geology, Government of Karnataka for providing the necessary data required for research and the anonymous reviewers for their valuable suggestions and comments.

Conflict of interest: The authors declare no conflict of interest.

\section{References}

[1] W. C. Burnett, H. Bokuniewicz, M. Huettel, W. S. Moore, and M. Taniguchi, "Groundwater and pore water inputs to the coastal zone," Biogeochemistry, vol. 66, no. 1-2, pp. 3-33, 2003. 
[2] M. Derbela and I. Nouiri, "Intelligent approach to predict future groundwater level based on artificial neural networks (ANN)," Euro-Mediterranean J. Environ. Integr., vol. 5, no. 3, pp. 1-11, 2020.

[3] A. Sharafati, S. B. H. S. Asadollah, and A. Neshat, "A new artificial intelligence strategy for predicting the groundwater level over the Rafsanjan aquifer in Iran," J. Hydrol., p. 125468, 2020.

[4] D. K. Todd and L. W. Mays, Groundwater hydrology. John Wiley \& Sons, 2004.

[5] M. P. Anderson, W. W. Woessner, and R. J. Hunt, "Applied groundwater modeling: Simulation of flow and advective transport," Acad. Press Inc., San Diego, CA. J. Hydrol., vol. 140, pp. 393-395, 1992.

[6] J. A. Oberdorfer, "Hydrogeologic modeling of submarine groundwater discharge: comparison to other quantitative methods," Biogeochemistry, vol. 66, no. 1-2, pp. 159-169, 2003.

[7] A. Malik, "Modelling groundwater level fluctuations in urban areas using artificial neural network," Groundw. Sustain. Dev., p. 100484, 2020.

[8] F. B. Banadkooki et al., "Enhancement of Groundwater-Level Prediction Using an Integrated Machine Learning Model Optimized by Whale Algorithm," Nat. Resour. Res., pp. 1-20, 2020.

[9] A. Seifi, M. Ehteram, V. P. Singh, and A. Mosavi, "Modeling and Uncertainty Analysis of Groundwater Level Using Six Evolutionary Optimization Algorithms Hybridized with ANFIS, SVM, and ANN," Sustainability, vol. 12, no. 10, p. 4023, 2020.

[10] A. K. Affandi and K. Watanabe, "Daily groundwater level fluctuation forecasting using soft computing technique," Nat. Sci., vol. 5, no. 2, pp. 1-10, 2007.

[11] A. Jalalkamali and N. Jalalkamali, "Groundwater modeling using hybrid of artificial neural network with genetic algorithm," African J. Agric. Res., vol. 6, no. 26, pp. 5775-5784, 2011.

[12] H. Yoon, S.-C. Jun, Y. Hyun, G.-O. Bae, and K.-K. Lee, “A comparative study of artificial neural networks and support vector machines for predicting groundwater levels in a coastal aquifer," $J$. Hydrol., vol. 396, no. 1-2, pp. 128-138, Jan. 2011, doi: 10.1016/j.jhydrol.2010.11.002.

[13] E. Fallah-Mehdipour, O. Bozorg Haddad, and M. A. Mariño, "Prediction and simulation of monthly groundwater levels by genetic programming,” J. Hydro-Environment Res., vol. 7, no. 4, pp. 253-260, 2013, doi: 10.1016/j.jher.2013.03.005.

[14] L. Tapak, A. R. Rahmani, and A. Moghimbeigi, "Prediction the groundwater level of hamadan-bahar plain, west of iran using support vector machines.," J. Res. Health Sci., vol. 14, no. 1, pp. 81-6, Jan. 2014, Accessed: Sep. 17, 2014. [Online]. Available: http://www.ncbi.nlm.nih.gov/pubmed/24402856.

[15] T. Van Ty and H. Van Hiep, "Groundwater level prediction using artificial neural networks: a case study in Tra Noc industrial zone, Can Tho city, Vietnam," J. Water Resour. Prot., vol. 10, no. 09, p. 870, 2018.

[16] Y. Z. Kaya, F. Üneş, M. Demirci, B. Taşar, and H. Varçin, "Groundwater level prediction using artificial neural network and M5 tree models," Aerul si Apa. Compon. ale Mediu., pp. 195-201, 2018.

[17] S. Lee, K.-K. Lee, and H. Yoon, "Using artificial neural network models for groundwater level forecasting and assessment of the relative impacts of influencing factors," Hydrogeol. J., vol. 27, no. 2, pp. 567-579, 2019.

[18] Y. Tang, C. Zang, Y. Wei, and M. Jiang, "Data-driven modeling of groundwater level with LeastSquare support vector machine and spatial-temporal analysis," Geotech. Geol. Eng., vol. 37, no. 3, pp. 1661-1670, 2019.

[19] N. Natarajan and C. Sudheer, "Groundwater level forecasting using soft computing techniques," Neural Comput. Appl., 2020, doi: 10.1007/s00521-019-04234-5.

[20] T. Roshni, M. K. Jha, and J. Drisya, "Neural network modeling for groundwater-level forecasting in coastal aquifers," Neural Comput. Appl., pp. 1-18, 2020.

[21] A. T. M. S. Rahman, T. Hosono, J. M. Quilty, J. Das, and A. Basak, "Multiscale Groundwater Level Forecasting: Coupling New Machine Learning Approaches with Wavelet Transforms," Adv. Water Resour., p. 103595, 2020.

[22] B. Yadav, P. K. Gupta, N. Patidar, and S. K. Himanshu, "Ensemble modelling framework for groundwater level prediction in urban areas of India," Sci. Total Environ., vol. 712, p. 135539, 2020, doi: 10.1016/j.scitotenv.2019.135539.

[23] N. S. Raghavendra and P. C. Deka, "Sustainable Development and Management of Groundwater Resources in Mining Affected Areas: A Review," Procedia Earth Planet. Sci., vol. 11, pp. 598-604, 2015, doi: 10.1016/j.proeps.2015.06.061.

[24] M. Buragohain and C. Mahanta, "A novel approach for ANFIS modelling based on full factorial design," Appl. Soft Comput., vol. 8, no. 1, pp. 609-625, 2008.

[25] G. Gallo, I. Perfilieva, M. Spagnuolo, and S. Spinello, "Geographical data analysis via mountain function,” Int. J. Intell. Syst., vol. 14, no. 4, pp. 359-373, 1999. 
[26] F. H. Fernández and F. H. Lozano, “GMDH Algorithm Implemented in Intelligent Identification of a Bioprocess," in ABCM Symposium series in Mechatronics, 2010, vol. 4, pp. 278-287.

[27] J. Xiao, H. Cao, X. Jiang, X. Gu, and L. Xie, "GMDH-based semi-supervised feature selection for customer classification,” Knowledge-Based Syst., vol. 132, pp. 236-248, 2017.

[28] I. Ebtehaj, H. Bonakdari, A. H. Zaji, H. Azimi, and F. Khoshbin, "GMDH-type neural network approach for modeling the discharge coefficient of rectangular sharp-crested side weirs," Eng. Sci. Technol. an Int. J., vol. 18, no. 4, pp. 746-757, 2015.

[29] D. Srinivasan, "Energy demand prediction using GMDH networks," Neurocomputing, vol. 72, no. 13, pp. 625-629, 2008.

[30] S. J. Farlow, Self-organizing methods in modeling: GMDH type algorithms, vol. 54. CrC Press, 1984.

[31] W. Liu et al., "Short-term load forecasting based on elastic net improved GMDH and difference degree weighting optimization," Appl. Sci., vol. 8, no. 9, p. 1603, 2018.

[32] I. Madala and A. G. Ivakhnenko, "Inductive Learning Algorithm for Complex System Modelling," CRC, Boca Raton, FL, 1994.

[33] R. E. Schapire, "The boosting approach to machine learning: An overview," in Nonlinear estimation and classification, Springer, 2003, pp. 149-171.

[34] J. H. Friedman, "Stochastic gradient boosting," Comput. Stat. Data Anal., vol. 38, no. 4, pp. 367-378, 2002.

[35] M. F. Li, X. P. Tang, W. Wu, and H. Bin Liu, "General models for estimating daily global solar radiation for different solar radiation zones in mainland China," Energy Convers. Manag., 2013, doi: 10.1016/j.enconman.2013.03.004.

[36] J. Nossent and W. Bauwens, "Application of a normalized Nash-Sutcliffe efficiency to improve the accuracy of the Sobol' sensitivity analysis of a hydrological model," Eguga, 2012.

[37] W. I. Gustafson and S. Yu, "Generalized approach for using unbiased symmetric metrics with negative values: Normalized mean bias factor and normalized mean absolute error factor," Atmos. Sci. Lett., 2012, doi: 10.1002/asl.393.

[38] H. Kling and H. Gupta, "On the development of regionalization relationships for lumped watershed models: The impact of ignoring sub-basin scale variability," J. Hydrol., vol. 373, no. 3-4, pp. 337-351, 2009, doi: 10.1016/j.jhydrol.2009.04.031.

[39] D. R. Legates and G. J. Mccabe, “A refined index of model performance: A rejoinder,” Int. J. Climatol., vol. 33 , no. 4 , pp. 1053-1056, 2013, doi: 10.1002/joc.3487. 